\title{
Maternal death and caesarean section in South Africa: Results from the 2011 - 2013 Saving Mothers Report of the National Committee for Confidential Enquiries into Maternal Deaths
}

\author{
G S Gebhardt, ${ }^{1} \mathrm{MB}$ ChB, MSc (Med Sci), MMed (O\&G), FCOG (SA); S Fawcus, ${ }^{2}$ MA, MB BCh, FRCOG; \\ J Moodley, ${ }^{3}$ MB ChB, FCOG, FRCOG, MD; Z Farina, ${ }^{4}$ MB ChB, DA (SA), FCA (SA); for the National Committee \\ for Confidential Enquiries into Maternal Deaths in South Africa \\ ${ }^{1}$ Department of Obstetrics and Gynaecology, Faculty of Medicine and Health Sciences, Stellenbosch University and Tygerberg Hospital, \\ Tygerberg, Cape Town, South Africa \\ ${ }^{2}$ Department of Obstetrics and Gynaecology, Faculty of Health Sciences, University of Cape Town and Mowbray Hospital, Cape Town, \\ South Africa \\ ${ }^{3}$ Department of Obstetrics and Gynaecology, Nelson Mandela School of Medicine, College of Health Sciences, University of KwaZulu-Natal, \\ Durban, South Africa \\ ${ }^{4}$ Department of Anaesthesia, Nelson Mandela School of Medicine, College of Health Sciences, University of KwaZulu-Natal, Durban, \\ South Africa, and Grey's Hospital, Pietermaritzburg, South Africa
}

Corresponding author: S Gebhardt (gsgeb@sun.ac.za)

\begin{abstract}
Background. In the latest (2011 - 2013) Saving Mothers report, the National Committee for Confidential Enquiries into Maternal Deaths in South Africa (SA) (NCCEMD) highlights the large number of maternal deaths associated with caesarean section (CS). The risk of a woman dying as a result of CS during the past triennium was almost three times that for vaginal delivery. Of all the mothers who died during or after a CS, $3.4 \%$ died during the procedure and $14.5 \%$ from haemorrhage afterwards. Including all cases of death from obstetric haemorrhage where a CS was done, there were 5.5 deaths from haemorrhage for every 10000 CSs performed.

Objective. To scrutinise the contribution or effect of the surgical procedure on the ultimate cause of death by a cross-cutting analysis of the 2011 - 2013 national data.

Methods. Data from the 2011 - 2013 triennial review were entered into an Excel database and analysed on a national and provincial basis. Results. There were 1243 maternal deaths where a CS was the mode of delivery and 1471 deaths after vaginal delivery. More mothers died as a result of CS in the provinces where there is a low overall CS rate. The following CS categories were identified as specific problems: bleeding during or after CS, pre-eclampsia and eclampsia, anaesthesia-related deaths, pregnancy-related sepsis and acute collapse and embolism.

Conclusion. This is an area of concern, and a concentrated effort should be done to make CS in SA safer. Several recommendations are made to this effect.
\end{abstract}

S Afr Med J 2015;105(4):287-291. DOI:10.7196/SAMJ.9351

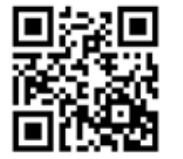

Worldwide, haemorrhage during pregnancy remains the most important cause of maternal death. ${ }^{[1]}$ As there are established practices and protocols to manage antepartum and postpartum haemorrhage $(\mathrm{PPH})$, failure to reduce deaths may in part be attributed to a rise in caesarean section (CS) rates with its increased surgical risks for bleeding. Major obstetric intervention by skilled healthcare workers should reduce maternal deaths. ${ }^{[2]}$

The National Committee for Confidential Enquiries into Maternal Deaths in South Africa (SA) (NCCEMD) has published triennial reports of maternal deaths ${ }^{[3]}$ since its inception in 1998. In the previous report, concern was raised about the increasing rate of haemorrhage during and after $\mathrm{CS}^{[4]}$ An extensive campaign to reduce these deaths was launched, with increased awareness, training and protocols. ${ }^{[5]} \mathrm{A}$ monograph on management of $\mathrm{PPH}^{[6]}$ as well as a manual of recommended techniques for safe $\mathrm{CS}^{[7]}$ were also produced. Despite this, in the latest report ${ }^{[8]}$ the NCCEMD again highlights the alarming rate of maternal deaths associated with CS. The most serious issue identified remains bleeding during or after
CS. Of all the deaths during or after a CS, one-third were due to hypovolaemic shock (as a final cause).

The risk of a woman dying during the past triennium as a result of CS was almost three times that for vaginal delivery: the case fatality rate (CFR), expressed as the number of deaths per 10000 causally related to mode of delivery, was 2.8 times higher for operative delivery (6.7/10 000 for vaginal births and 18.9/10 000 for CS).

There are limitations in using fatality rates for CS, as it is difficult to separate the inherent risk of the condition for which the surgery was done (such as eclampsia or life-threatening bleeding from placenta praevia) from the risk associated with the procedure itself (surgical, anaesthesia or postoperative care). During 2011 - 2013, of all the CSs performed in public sector facilities, 35\% took place at district hospitals, $40 \%$ at regional hospitals and $25 \%$ at tertiary or central hospitals, which is similar to previous years.

Of the 1243 mothers who died during or after a CS, $42(3.4 \%$ of all CS deaths) died as a result of bleeding problems during the procedure, and 180 (14.5\% of all CS deaths) from haemorrhage following the procedure. Including all other cases of death from 
obstetric haemorrhage (e.g. abruptio placentae and placenta praevia) where a CS was done $(N=363)$, the haemorrhage CFR equates to 5.5 deaths from haemorrhage for every 10000 CSs performed.

\section{Objectives}

The triennial data are analysed and organised into categories according to the major causes of maternal deaths; each category is reviewed by an expert in the field. As there were CS deliveries in all these separate clusters, the aim of this cross-cutting analysis of the 2011 - 2013 national data was to scrutinise the contribution or effect of the surgical procedure on the ultimate cause of death.

\section{Methods}

Data from the completed 2011-2013 triennial review were entered into an Excel database and analysed on a national and provincial basis. Data are anonymous and collected as part of the NCCEMD process. Permission to publish the information was obtained from the National Department of Health.

\section{Results}

There were 2831066 deliveries in SA during the past triennium (2011 - 2013), of which 655686 were by CS, amounting to a national CS rate of $23.1 \%$. There were 1243 maternal deaths where a CS was the mode of delivery and 1471 deaths after vaginal delivery. Most deliveries took place in Gauteng Province, and most CSs (in terms of actual numbers) were done in KwaZulu-Natal Province (KZN).

There was a striking difference in CS rates between the provinces, with the highest CS rate $(28.8 \%)$ in KZN and the lowest $(15.4 \%)$ in Limpopo (Fig. 1).

The CS CFR compared with the number of CS deaths per province is shown in Table 1. There were five provinces (Eastern Cape, Free State, North West, Mpumalanga and Limpopo) in which the CFR was higher than the national average.

When the CS rate for each province was compared with its CFR from CS, more mothers died from CS in the provinces with a low overall CS rate. The provinces with a CS rate of $>23 \%$ all had a CS CFR of $<20$, and those with a CS rate of $<17 \%$ had the worst CFR (Fig. 2). The negative correlation between the CS rate and the CFR is shown in Fig. 3.

Table 2 shows the relationship between primary obstetric cause of death and route of delivery, and includes those patients undelivered at time of death. It includes the delivery type-specific CFR and separates patients identified as bleeding during or after CS (BDACS) from the overall haemorrhage group to show its rank in terms of the other deaths (sorted in descending order). It also shows the relative risk (RR) of dying according to type of delivery.

As a final cause of death, hypovolaemic shock was associated with one-third of all CS deaths; this risk was almost five times higher than for vaginal delivery. Likewise, the risk of dying from acute collapse due to embolism was 4.5 times increased after CS.

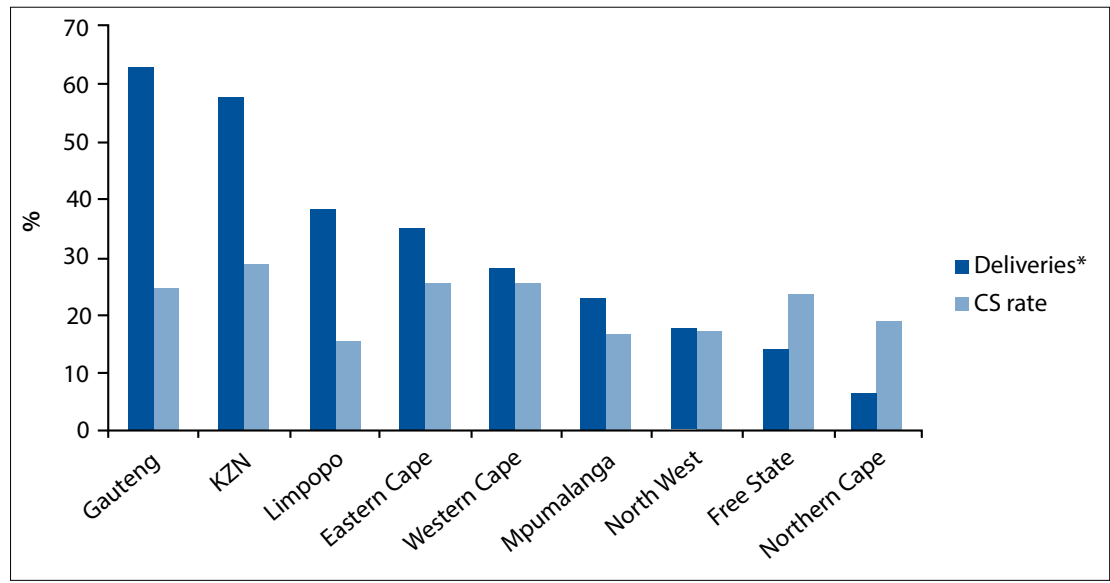

Fig. 1. CS rates per province (\%), $2011-2013$, with the total number of deliveries $\left({ }^{*}\right.$ deliveries presented as total number $\times 10000)$.

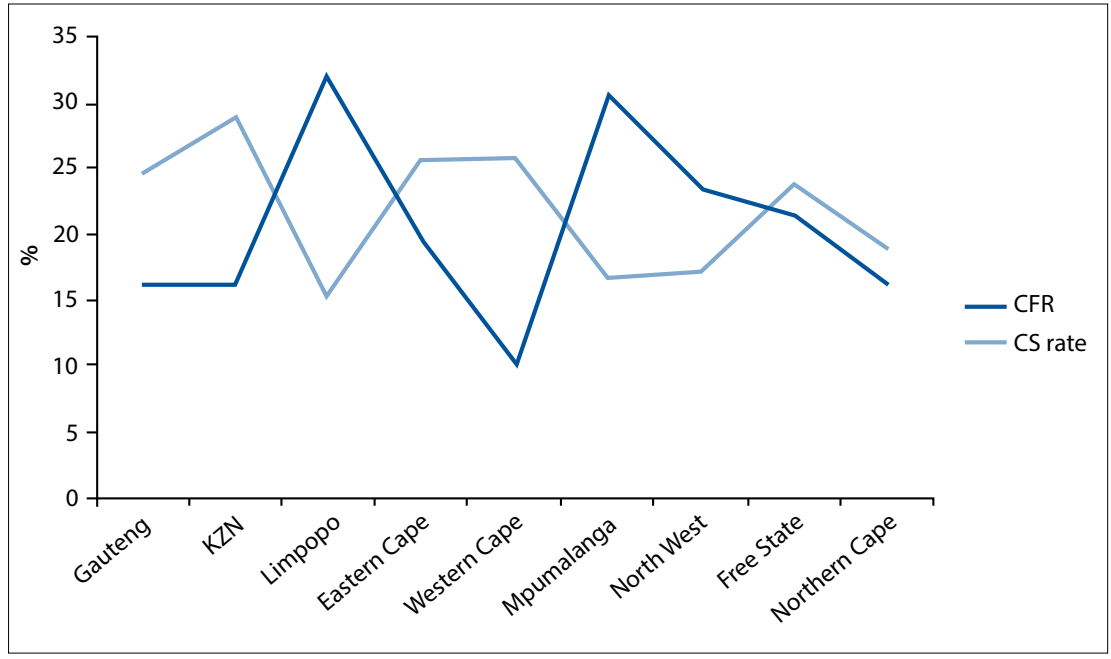

Fig. 2. Comparison of CS rate (\%) and CFR (/10 000 CSs) from CS per province, 2011 - 2013.

Table 1. Comparison of CS deaths per province, 2011 - 2013, proportion of the total deaths in each province, and CFRs

\begin{tabular}{llll}
\hline Province & $\begin{array}{l}\text { Deaths during } \\
\text { or after CS, } \boldsymbol{n}\end{array}$ & $\begin{array}{l}\text { Proportion of total deaths } \\
\text { for the province, \% }\end{array}$ & CFR, /10 000 CSs \\
\hline Western Cape & 73 & 34.1 & 10.1 \\
Gauteng & 253 & 29.8 & 16.4 \\
KZN & 273 & 28.3 & 16.4 \\
Northern Cape & 20 & 18.2 & 16.5 \\
South Africa & $\mathbf{1 2 4 3}$ & $\mathbf{2 7 . 9}$ & $\mathbf{1 8 . 9}$ \\
Eastern Cape & 174 & 29.3 & 19.5 \\
Free State & 74 & 26.3 & 21.6 \\
North West & 71 & 24.3 & 23.5 \\
Mpumalanga & 117 & 29.3 & 30.5 \\
Limpopo & 188 & 25.1 & 31.9
\end{tabular}


As can be deduced from the data in Table 2 death of patients with CS as route of delivery was a specific problem in the following categories:

- Bleeding during or after CS
- Pre-eclampsia and eclampsia (six times increased risk of dying)

- Anaesthesia-related deaths

- Pregnancy-related sepsis (three times increased risk)

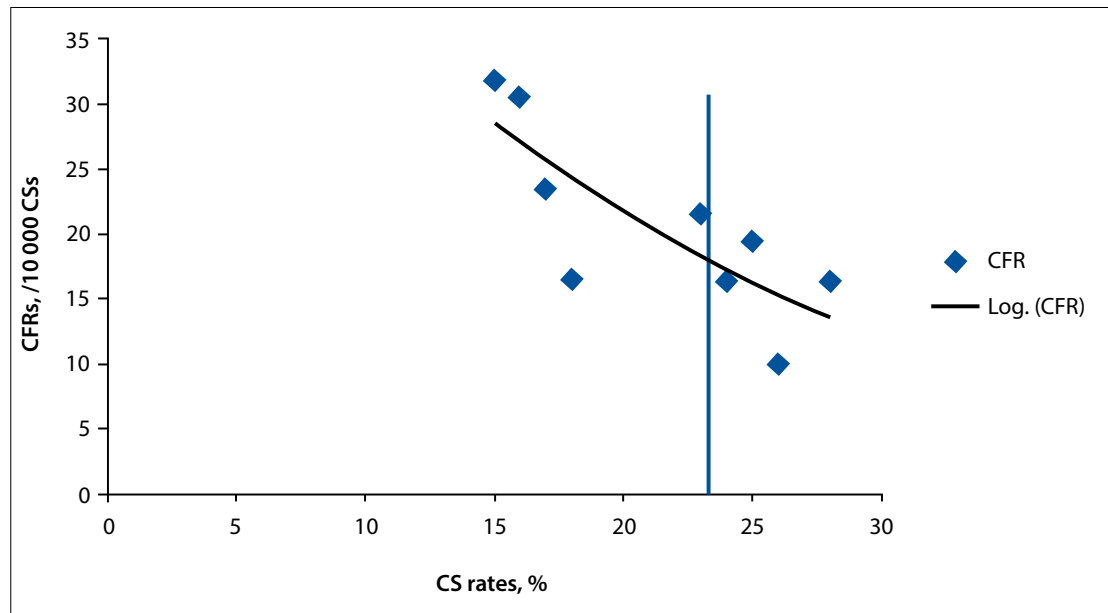

Fig. 3. Graphical presentation of negative correlation between CS rates (\%) and CFRs (/10 000 CSs) in provinces, $2011-2013$.
- Acute collapse and embolism (combined, a five times increased risk).

Even though the indication for the CS may have been an attempt to save a life, this is an area of concern and a concentrated effort is necessary to make these CSs safer.

\section{Bleeding during or after CS}

BDACS was linked to 222 deaths in 2011 2013. In previous reports it was linked to 78 deaths in 2002 - 2004, 141 deaths in 2005 2007, and 180 deaths in 2008 - 2010. Since the 2008 - 2010 report, the category 'other uterine trauma' (which was used in previous years, and was a composite of bleeding with CS as well as vaginal/cervical trauma) was changed so that BDACS is measured separately. This category excludes deaths from haemorrhage attributed to other pathology (placenta praevia, abruptio placentae, cervical tears, bowel perforation during CS, etc.) of patients also delivered by CS. There may be some

Table 2. Relationship between primary obstetric cause of death and route of delivery

\begin{tabular}{|c|c|c|c|c|c|}
\hline & $\begin{array}{l}\text { Vaginal } \\
\text { deliveries, } n\end{array}$ & $\begin{array}{l}\text { CFR for vaginal delivery only, } \\
\text { /10 } 000 \text { vaginal deliveries }\end{array}$ & CSs, $n$ & $\begin{array}{l}\text { CFR for CS } \\
\text { delivery only, } \\
\text { /10 } 000 \mathrm{CSs}\end{array}$ & $\begin{array}{l}\text { RR (for CS delivery, if RR } \\
\text { for vaginal delivery }=1 \text { ) }\end{array}$ \\
\hline \multicolumn{6}{|l|}{ Primary obstetric cause of death } \\
\hline Non-pregnancy-related infections & 646 & 2.9 & 166 & 2.5 & \\
\hline Hypertension & 154 & 0.7 & 272 & 4.1 & 5.9 \\
\hline Pre-eclampsia & 39 & 0.18 & 68 & 1 & 5.8 \\
\hline Eclampsia & 78 & 0.36 & 146 & 2.2 & 6.2 \\
\hline HELLP syndrome & 27 & 0.4 & 44 & 0.67 & 1.6 \\
\hline Medical and surgical disorders & 165 & 0.76 & 125 & 1.9 & 2.5 \\
\hline Obstetric haemorrhage (excludes BDACS) & 250 & 1.1 & 147 & 2.2 & 1.9 \\
\hline Pregnancy-related sepsis & 112 & 0.5 & 100 & 1.5 & 2.96 \\
\hline Bleeding - CS (BDACS) & & & 222 & 3.3 & \\
\hline Miscarriage & 6 & 0.027 & 1 & 0.01 & \\
\hline Unknown & 72 & 0.3 & 41 & 0.6 & \\
\hline Coincidental cause & 14 & 0.06 & 20 & 0.03 & \\
\hline Anaesthetic complications & 5 & 0.02 & 79 & 1.2 & \\
\hline Embolism & 23 & 0.1 & 44 & 0.6 & 6.3 \\
\hline Acute collapse - cause unknown & 24 & 0.1 & 27 & 0.4 & 3.7 \\
\hline \multicolumn{6}{|l|}{ Final cause of death } \\
\hline Circulatory system & 486 & & 567 & & \\
\hline Hypovolaemic shock & 279 & & 407 & & 4.8 \\
\hline Septic shock & 207 & & 160 & & \\
\hline Respiratory failure & 595 & & 282 & & \\
\hline Cardiac failure & 298 & & 363 & & \\
\hline Pulmonary oedema & 113 & & 133 & & \\
\hline Cardiac arrest & 185 & & 230 & & \\
\hline Acute collapse due to embolism & 38 & & 52 & & 4.5 \\
\hline
\end{tabular}


overlap, as death from an atonic uterus during CS may be classified under atony or under BDACS. The BDACS group is (in terms of numbers) as important a cause of death as pregnancy-related sepsis.

There were 363 deaths associated with haemorrhage and CS, of which 222 were directly associated with the procedure; a further 54 CS-related deaths in the haemorrhage group were attributed to abruptio placentae and 26 to complications arising from placenta praevia or placenta accreta. A total of 110 deaths (for any method of delivery) were attributed to abruptio placentae, with the risk of dying from abruptio placentae being relatively low (1/25 736 deliveries), but more than eight times increased when abruptio placentae was managed with a CS (RR 8.8; 95\% confidence interval 5.5 - 13; $p<0.0001$ )

Deaths were assessed as clearly avoidable in $70 \%$ of cases of bleeding during CS and in $72 \%$ of cases of bleeding after CS; only $1.8 \%$ of women who died were assessed as having no suboptimal care. The greatest problem was at district hospital level.

The following themes arose from the folder assessments of the BDACS cases:

- The source of the BDACS was not always specified in the patient folder, i.e. whether it was due to uterine atony, traumatic extension of uterine incision or tears, or placental site bleeding.

- Many women died in the ambulance before during or after referral. This reflected reluctance to perform necessary surgery at the district hospitals (due to lack of sufficient blood products and lack of surgical competence), but also ambulance delays.

- Better monitoring by nurses was observed compared with previous years, but there was poor response to deteriorating vital signs by nurses and doctors, and the latter frequently gave telephonic advice rather than assessing the patient. Colour-coded early-warning charts were not found in the folders.

- Poor use of uterotonics, especially ergometrine to prevent and treat uterine atony at CS

- Poor surgical skills at initial CS

- Poor interaction between the surgical and anaesthetic teams at all stages of the operative process:

- Preoperative evaluation of the patient and anticipation of potential problems

- Communication during the surgical procedure with regard to changes in the condition of the patient and the operative situation

- Postoperative management and care plans.
- Referral for post-CS bleeding was arranged, rather than a relook laparotomy.

- Internal bleeding was common in post-CS bleeding deaths, but there was a delay in recognition.

- Poor use of uterine compression sutures, balloon tamponade and uterine tourniquets.

\section{Anaesthesia deaths and frequency of CS}

The percentages of women dying from anaesthetic complications per province, and the CS rate per province, are shown in Fig. 4. It can again be deduced that the anaesthetic death rate is negatively correlated with the CS rate - the risk of dying from anaesthetic complications is higher when the CS rate is lower. Most deaths arising from complications from anaesthesia were in Limpopo, Mpumalanga and the Eastern Cape.

The deaths from haemorrhage in particular reveal the issue of poor interaction with the surgical and anaesthetic teams, where volumes of blood loss, severity of hypotension and degree of compromise of the patient were not appreciated across the entire surgical team. Patients were transferred to the ward from the recovery room in a compromised state with no clear plan of monitoring or intervention.

\section{Discussion and}

\section{recommendations}

Based on the data, the following recommendations are made:

A. To reduce deaths from bleeding during or after CS:

- Prevent anaemia, prolonged labour and second-stage CS as far as possible.
- Hospital managers should ensure that a continuous stock of emergency blood and freeze-dried plasma be available at district hospitals.

- Implement the use of standard protocols for the use of uterotonics for prevention and/or management of bleeding after CS.

- Training to focus on problem recognition and skills training of doctors performing CS, and anaesthetics for obstetric patients at district hospitals.

- Women with abnormal vital signs after CS should not leave the recovery area and should not be transferred to a postnatal ward in an unstable condition; a doctor should come and assess such a patient immediately.

- There should be a clear plan for regular planned reassessment of at-risk women who are deemed suitable for transfer to the postoperative ward, and written instructions should be sent to the ward with regard to targets at which earlier medical review should be requested.

- All women with blood loss after CS need to be resuscitated immediately and a doctor called to assess them, with immediate return to theatre if response to uterotonics is poor.

- Direct telephonic links should be provided for 24-hour specialist support to district hospital doctors.

- Emergency transport to be onsite for transfers from district hospitals.

- Essential skill competencies to include: district hospitals - safe CS, safe anaesthesia, balloon tamponade, uterine compression sutures and uterine tourniquet; and regional/tertiary hospitals - all the above plus hysterectomy.

- For second-stage CS, with the head impacted in the pelvis, the risk of lateral tears and blood loss is lower with the reverse breech extraction method of delivery of the baby. ${ }^{[9]}$

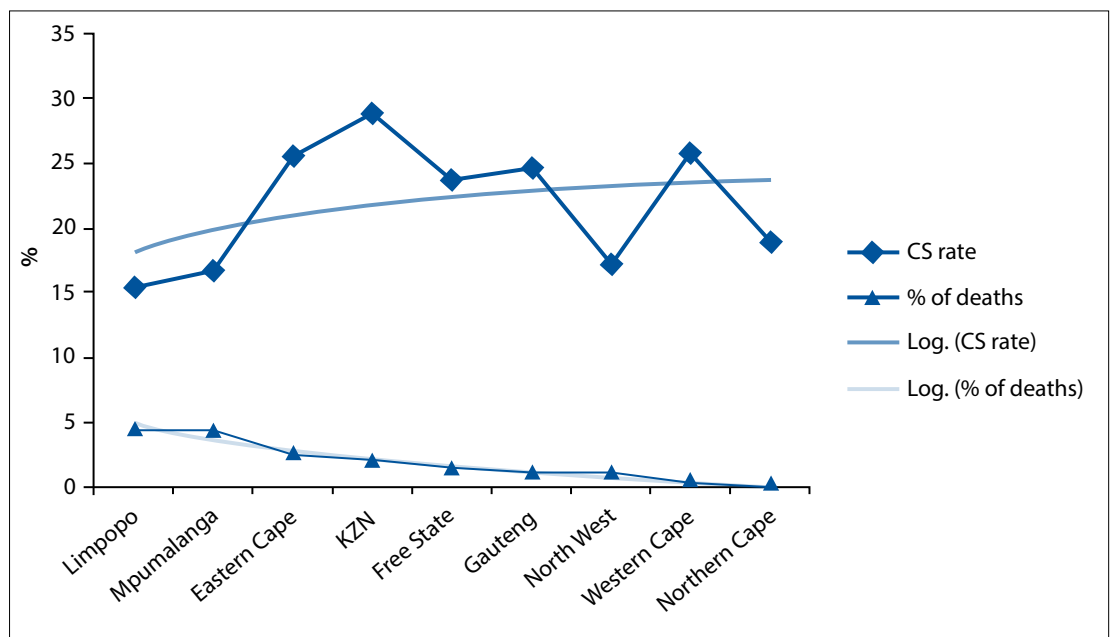

Fig. 4. Percentage of women dying from anaesthetic complications per province, and CS rate (\%) per province. A logarithmic trend line was added. 
B. To reduce deaths from CS in hypertensive conditions:

- All women with eclampsia, irrespective of type, must be managed at regional/tertiary levels of care. Aim for a vaginal delivery as far as possible.

- Eclamptic women with a Glasgow Coma Score (GCS) of 13 - 15/15 generally do well, and if a CS is required, regional anaesthesia can be considered as long as:

- This is carried out by an experienced anaesthetist or supervised by such a person

- The high blood pressure is stabilised (with several readings) prior to anaesthesia

- There is no coagulation problem, the platelet count is $>70 \times 10^{9} / \mathrm{L}$, and there is no evidence (clinical or biochemical) of the haemolysis/ elevated liver enzymes/low platelets (HELLP) syndrome

- Preventive measures are taken against PPH and the patient is observed after the procedure in a high-care bed or one dedicated for this purpose for at least 24 hours after the initiation of magnesium sulphate.

- In the case of eclamptic women with a GCS of $10-12 / 15$, if a CS is required, this should be done under general anaesthesia by an experienced anaesthetist or under his/her supervision.

- The oedematous eclamptic may have a swollen airway. In addition, if such patients have an elevated serum urate level, they may be in danger of pulmonary oedema. Careful attention must be given to fluid balance management.

- The restless eclamptic - these patients may be hypoxic and/or have cerebral oedema.

- Strong consideration must be given to ventilate such patients (oedematous or restless eclamptics) for at least 24 hours following delivery.

- Patients with a GCS $\leq 9$ should be ventilated for at least 24 hours after the procedure.

- The pressor response to intubation can be exacerbated in preeclamptic patients. High blood pressure should be controlled, even in the operating theatre before intubation. An expert familiar with the use of agents (such as magnesium sulphate and alfentanil) to facilitate safe intubation should be contacted.

- Post-delivery care is mandatory in all pre-eclamptics, and a stepwise decrease in the dosage of antihypertensives may prevent readmission due to hypertensive complications.

- All CSs should be performed by experienced persons and steps taken to minimise bleeding.

C. To reduce deaths from acute collapse and embolism following CS: - Measure the body mass index (BMI, $\left.\mathrm{kg} / \mathrm{m}^{2}\right)$ at booking. Women with a booking BMI of $\geq 40$ should preferably be managed at a specialist/ regional hospital level owing to the increased risk of thrombosis, diabetes, macrosomic babies, difficult CS (both anaesthesia and surgery) and $\mathrm{PPH}$. Women with a booking BMI of $\geq 50$ should preferably deliver at a tertiary hospital owing to increased anaesthetic risks, including difficult airway and postoperative difficulty in breathing.

- The Saving Mothers report of 2005 - 2007 ${ }^{[10]}$ included protocols on the risk stratification of pregnant women for thromboembolism. This (or Green-top Guideline No. 37a from the Royal College of Obstetricians and Gynaecologists ${ }^{[11]}$ ) should be used to identify women at risk of thromboembolism, and provide effective thromboprophylaxis until 7 days after delivery.

\section{To reduce deaths from post-CS sepsis:}

- Adhere to the accepted antisepsis strategies during surgery.

- Use the World Health Organization surgical safety checklist (maternity version) as published in the 5th Saving Mothers report. ${ }^{[4]}$
Administer prophylactic antibiotics (a first-generation cephalosporin, e.g. $1 \mathrm{~g}$ cefazolin intravenously (IV)) to EVERY woman who has a CS, whether elective or emergency. Administer 30 - 60 minutes before surgery (as premedication). For women with severe penicillin allergy, clindamycin or erythromycin can be used instead.

- Give a higher dose (2 g) when the BMI is $>30$ (or the patient weighs $>100 \mathrm{~kg}) \cdot{ }^{[12]}$

- Although there is no good evidence from randomised trials, the following women may benefit from a prolonged course (3 days) of antibiotics (e.g. ampicillin 2 g 6-hourly, gentamycin $240 \mathrm{mg}$ IV daily, and metronidazole $400 \mathrm{mg}$ 8-hourly orally):

- HIV-positive patients

- Blood transfusion during surgery

- Blood loss >1 $000 \mathrm{~mL}$ during surgery

- Second-stage emergency CS

- Prolonged (>12 hours) rupture of membranes

- $>5$ vaginal examinations during labour

- When a birth attendant had to push up the head vaginally during difficult delivery of the head.

- Anticipate difficult surgery (specifically two or more previous CSs, BMI >40, previous CS with septic wound, second-stage $\mathrm{CS}$ ) and request the most experienced surgeon to operate. Do a longitudinal abdominal incision for CS in patients with two or more previous Pfannenstiel incisions, especially for an emergency $\mathrm{CS}$, to prevent accidental bowel or bladder injury.

\section{Conclusion}

The inverse relationship between CS deaths and the CS rate in specific provinces could be attributed to a variety of reasons, including lack of easy access to safe CS, long waiting times to get to theatre and retention of skills when a reasonable CS rate is maintained. What it does argue is that doing more CSs does not necessarily lead to more surgically related deaths.

A concentrated effort should be made by everyone involved in all the various aspects of surgical delivery to decrease the number of deaths associated with the procedure. Medical students are illequipped to do surgery after graduation, yet they are expected to do a major invasive procedure in women with complex, altered physiology where complications that can challenge even a highly skilled doctor can arise within seconds. Could CS skills training not be incorporated into the undergraduate medical curriculum?

\section{References}

1. Say L, Chou D, Gemmill A, Tunçalp Ö, et al. Global causes of maternal death: A WHO systematic analysis. Lancet Glob Health 2014;2(6):e323-e333. [http://dx.doi.org/10.1016/S2214$109 \mathrm{X}(14) 70227-\mathrm{X}]$

2. Weil O, Fernandez H. Is safe motherhood an orphan initiative? Lancet 1999;354(9182):940-943. [http://dx.doi.org/10.1016/S0140-6736(99)02369-7]

3. Moodley J, Pattinson RC, Fawcus S, et al. The confidential enquiry into maternal deaths in South Africa: A case study. BJOG 2014;121(Suppl 4):53-60. [http://dx.doi.org/10.1111/1471-0528.12869]

4. Pattinson RC, ed. Saving Mothers: Fifth Report on Confidential Enquiries into Maternal Deaths in South Africa 2008 - 2010. Pretoria: National Department of Health, 2012.

5. Fawcus S, Moodley J. Haemorrhage associated with caesarean section in South Africa - be aware. S Afr Med J 2011;101(5):306,308-309.

6. Fawcus S, Moodley J. A Monograph of the Management of Postpartum Haemorrhage. Pretoria: Department of Health, 2011.

7. Moodley J, ed., National Department of Health. A Monograph on Caesarean Section. Pretoria: Department of Health, 2013.

8. Pattinson RC, ed. Saving Mothers 2011-2013: The Sixth Report of the National Committee for Confidential Enquiries into Maternal Deaths in South Africa. Pretoria: Government Printer, 2014.

9. Berhan Y, Berhan A. A meta-analysis of reverse breech extraction to deliver a deeply impacted head . Berhan Y, Berhan A. A meta-analysis of reverse breech extraction to deliver a deeply impacted head
during cesarean delivery. Int J Gynaecol Obstet 2014;124(2):99-105. [http://dx.doi.org/10.1016/j. ijgo.2013.08.014]

10. Pattinson RC, ed. Saving Mothers: Fourth Report on Confidential Enquiries into Maternal Deaths in South Africa 2005 - 2007. Pretoria: National Department of Health, 2010.

11. Royal College of Obstetricians and Gynaecologists. Thrombosis and Embolism during Pregnancy and the Puerperium, Reducing the Risk (Green-top Guideline No. 37a). London: RCOG, 2010. https:// www.rcog.org.uk/en/guidelines-research-services/guidelines/gtg37a/ (assessed 5 January 2015).

12. American College of Obstetricians and Gynecologists. Use of prophylactic antibiotics in labor and delivery. Practice Bulletin No. 120. Obstet Gynecol 2011;117(6):1472-1483. [http://dx.doi.org/10.1097/ aog.0b013e3182238c31

Accepted 15 January 2015. 